\title{
El salario de las trabajadoras domésticas en Argentina, 1908-1956
}

Female domestic workers wages in Argentina, 1908-1956

\author{
Mateo AlleRAND* \\ Universidad de Buenos Aires. Facultad de Ciencias \\ Económicas. Departamento de Humanidades. Centro \\ de Estudios Económicos de la Empresa y el Desarrollo \\ (CEEED). Buenos Aires, Argentina. \\ ORCID: 0000-0003-0126-4713
}

Eduardo Martín Cuesta**

Grupo de estudios Urbanos y Generacionales, CONICET-Universidad de Buenos Aires. Instituto

Interdisciplinario de Economía Política de Buenos Aires.

Buenos Aires, Argentina.

ORCID: 0000-0002-2017-2278

\author{
ERNESTO CURVALE ${ }^{* * *}$ \\ Universidad de Buenos Aires. Facultad de Ciencias \\ Económicas. Departamento de Humanidades. Centro \\ de Estudios Económicos de la Empresa y el Desarrollo \\ (CEEED). Buenos Aires, Argentina. \\ ORCID: 0000-0001-7623-8363
}

Recibido: $17 / 07 / 2020$

Aceptado: 03/09/2020

doi: https://doi.org/10.20318/femeris.2020.5762

\begin{abstract}
Resumen. Los estudios de historia y género han avanzado en los últimos cincuenta años. Con mayor o menor detalle, las investigaciones con abordaje de género también han profundizado en la historia económica de este tipo de desigualdad. En el caso de Argentina, se cuenta con excelentes producciones acerca de las mujeres en el mundo laboral. Entre otros puntos, sobre el carácter invisible del trabajo doméstico, no remunerado, así como sobre las trabajadoras domésticas. En este aspecto, todavía hay una vacancia acerca de conocer los ingresos "formales" de las trabajadoras del sector. Por ello, en este trabajo se presenta y analiza la evolución de los salarios de las trabajadoras domésticas en la ciudad de Buenos Aires en el largo plazo. Para ello, se utilizan nuevas y diferentes fuentes que permiten construir una serie continua y homogénea de salarios de mujeres trabajadoras del servicio doméstico, que se analizan en conjunto con otros indicadores como la población, el PBI y el desempleo. El análisis conduce a entender el comportamiento de estos salarios en el contexto de la actividad económica local y
\end{abstract}

\footnotetext{
*mateoallerand@gmail.com

${ }^{* *}$ cuesta@filo.uba.ar

*** ernestocurvale@gmail.com
} 
del país. Esto permite observar el impacto de los ciclos y coyunturas económicas en las trabajadoras del sector, así como su devenir en el largo plazo.

Palabras clave: trabajadoras domésticas, salarios, género, Argentina, Buenos Aires.

Abstract. The studies of history and gender inequalities were increased in the lasts fifty years. In the Argentine's case, there are excellent researches about workers women. Also, there are good studies about the invisibility of domestic labour, unpaid, as well as domestic workers. In this aspect, there is still a vacancy about knowing the female workers at domestic service. Therefore, this works presents and analyses the evolution of the salaries of female domestic workers in Buenos Aires city in the long term. We use different sources that allow building continuous and homogeneous series. That is analysed with other relevant economic indicators such as GDP and unemployment. These allow us to observe the impact of the cycles and economic context in the domestic service female workers in the short and long term.

Keywords: female domestic workers, salaries, gender, Argentina, Buenos Aires.

\section{Introducción}

El trabajo femenino es un tema de agenda en las investigaciones, tanto para el presente como el pasado. De allí que el mundo laboral de la Argentina cuenta con numerosos estudios sobre los salarios de los trabajadores desde el siglo XIX a la actualidad. En particular, se ha avanzado en conocer el trabajo femenino, quedando aún mucho por recorrer; por ejemplo, aún se conoce poco acerca de las trabajadoras del servicio doméstico. Este sector cobra particular relevancia por la importante presencia de mano de obra femenina.

Esta notable ausencia cobra sentido en un contexto en el cual los trabajos con mano de obra mayoritariamente femenina en general, y el servicio doméstico en particular, han sido poco investigados. Las tareas de cuidado ocupan un lugar subordinado dentro del mercado laboral, lo que se evidencia en menores salarios. Asimismo, las tareas domésticas realizadas todos los días en cada hogar son dejadas de lado en la economía "formal", al extremo de no ser incluidas como actividad en el sistema de cuentas nacionales.

En este trabajo, se presentará y analizará la evolución de los salarios de las trabajadoras del servicio doméstico en Buenos Aires, desde inicios hasta mediados del siglo XX. En primer lugar, se hará un breve análisis de la historiografía sobre el tema. Luego, se presentarán las fuentes y metodología utilizadas. En tercer lugar, se presentarán y analizarán los salarios de las trabajadoras domésticas, en el contexto económico local. El trabajo cierra con unas consideraciones finales y posibles líneas de investigación futuras.

\section{Estado de la cuestión}

Hace más de dos décadas que se incrementa el interés de los investigadores en economía, y en historia económica, sobre los niveles de vida, la desigualdad y su relación con el crecimiento y el desarrollo, además del impacto de las políticas económicas ${ }^{1}$.

\footnotetext{
${ }^{1}$ En el caso de Argentina, con el aporte de antiguos y nuevos datos, pero con nuevos interrogantes, la historiografía económica reciente posee una nueva agenda, dentro de la cual está entender y analizar la evolución y características de 
En particular, se entiende el concepto de desigualdad en economía desde el punto de vista del consumo, del gasto, o del ingreso. Dentro del ingreso, el foco se ha puesto en el salario; las diferencias salariales se entienden como originadas por la capacitación (skill premium), por tareas (obreros y empleados administrativos - cuello blanco o azul), por sector industrial (intra o inter sectorial), y más recientemente, por género.

Estos diferenciales se asignan a características del mercado de trabajo, ya sea por políticas comerciales (el libre comercio incentivaría la demanda de ciertos productos en desmedro de otros, afectando el trabajo en ciertos sectores) o por características propias del mercado de trabajo (oferta y demanda). También, se observa el impacto en la productividad de la mano de obra ${ }^{2}$, el nivel de capacitación (y sus incentivos), y el nivel educativo (y de escolarización). Esto también guarda estrecha relación con el cambio tecnológico 3 .

Asimismo, se ha puesto en consideración el contexto histórico y la relación entre economías con diferente grado de desarrollo. Por ejemplo, se ha señalado que el impacto de estos cambios es diferente según se trate de economías en desarrollo, desarrolladas o pobres.

Por otro lado, los procesos de Industrialización Sustitutiva de Importaciones (ISI) de los países en desarrollo durante parte del siglo XX también impactan en la evolución de las diferencias salariales ${ }^{4}$. En el mismo sentido, el proceso de globalización y/o del libre comercio, se entiende como base de los cambios en los diferenciales. Desde otros puntos de vista, también se interpreta que las políticas públicas son definitorias en la evolución de los diferenciales salariales: regulaciones de los salarios, nivel de empleo público, poder sindical, los subsidios (y consecuentemente los comportamientos rent-seekers), etc.

En la teoría económica, se suele abordar de manera tradicional la desigualdad salarial desde la perspectiva de la demanda en base a dos criterios (Esquivel, 2007): discriminación (diferentes salarios por el mismo trabajo/tarea) o segregación (diferentes trabajos y salarios según grupo o género $)^{5}$. Para el caso de la discriminación en general, el método tradicional de análisis ${ }^{6}$ es entender las causas de diferentes salarios por motivos "explicables" (justificables ${ }^{7}$ ) o por motivos "no explicados". La discriminación "pura" sería el segundo caso, la segregación ${ }^{8}$ se puede entender como intersectorial (horizontal) u

las ratios de bienestar, la desigualdad, y también las diferencias regionales (Hora, 2007) (Gelman, 2011). Estas nuevas perspectivas incluyen como uno de sus intereses establecer comparaciones no sólo a nivel internacional, sino también a nivel del interior de los países. En este sentido en los últimos años se han desarrollado trabajos que buscan comprender las diferencias, similitudes, convergencias y divergencias entre las ratios de bienestar y las economías de diferentes regiones de la Argentina.

${ }^{2}$ Se recomienda ver los trabajos de Camou y Maubrigades (2006).

${ }^{3}$ Ver Acemoglu (2002) o Goldin (2007).

${ }^{4}$ Todavía falta trabajar en profundidad en un abordaje desde el concepto de centro-periferia.

${ }^{5}$ Ver Becker (1971) y Altonji y Blank (1999). Según Polacheck (1995), las explicaciones por el lado de la demanda sobre segregación ocupacional son anteriores a los primeros estudios de diferenciales de salarios por género desde una perspectiva de la oferta. Este último enfoque entiende diferentes preferencias acerca de horarios, flexibilidad, etc. Por ello, las diferencias salariales serían resultado de decisiones personales.

${ }^{6}$ Para casos de estos abordajes, ver (Blinder, 1973) (Oxxaca, 1973). Una crítica al abordaje, en Bergmann (2004).

${ }^{7}$ Esto supone entender que las diferencias salariales están dadas por características intrínsecas, lo cual es discutible. Para profundizar este problema ver Bergmann (2004).

${ }^{8}$ Para un análisis sobre la segregación, véase Goldin (1990). 
ocupacional (vertical ${ }^{9}$ ). También se ha relacionado con la globalización, el crecimiento y el desarrollo económico, sea como causa o consecuencia.

Desde un principio, en los trabajos sobre género, se reconoció la existencia de una evolución en la participación de las mujeres en el mercado de trabajo, en relación con el contexto histórico, que en parte explicaría el diferencial. Según algunas interpretaciones, en el primer cuarto del siglo XX la participación de las mujeres en el mundo del trabajo era importante; luego descendió hasta mediados de siglo y de allí en adelante comenzó a crecer. Es el modelo de la "U" (Goldin, 1990), que para el caso de Argentina ha sido discutido, tomando en cuenta otros elementos, como que el ingreso al mercado laboral podría estar relacionado con la concentración de capital en cada sector industrial (Rocchi, 2001).

Lo hasta aquí presentado permite pensar que los enfoques económicos son insuficientes para comprender toda la magnitud y complejidad de la discriminación salarial por género. El trasfondo es una sociedad burguesa patriarcal, y sistemas sociales, políticos y culturales donde la discriminación de género está muy arraigada y naturalizada ${ }^{10}$.

En este sentido, Esquivel (2007) hace énfasis en las dificultades que enfrentan las mujeres para acceder a ciertos sectores del mercado laboral, así como la imposibilidad de alcanzar puestos jerárquicos. Estos límites vedan el paso de las mujeres hacia ciertas tareas, así como también "feminizan" otras, como las de atención doméstica. Lo cual nos coloca en el tema del trabajo doméstico, la mirada de la economía feminista en relación al tema, tanto de D’Alessandro (2016) como de Rodríguez Enríquez (2017), es clara al respecto. El servicio doméstico (remunerado y no remunerado) es un claro ejemplo de las desiguales relaciones de género que atraviesan nuestra sociedad. Pérez Orozco (2014) coloca en el centro de la discusión la desigual distribución de las tareas de cuidado, no solo entre géneros, sino también entre los distintos países, como la posición subordinada que estas actividades ocupan en el mercado laboral en términos de remuneración y de protección social.

Acerca de las trabajadoras en Argentina durante el período existe una historiografía importante. Entre los antecedentes, se pueden citar los trabajos de Spalding (1970) y Guy (1981). Trabajos más recientes, en línea con la historiografía internacional, han abordado el tema en profundidad, como los casos de Barrancos (2007) y Lobato (2007). Sobre el espacio de la ciudad de Buenos Aires, Queirolo (2006) (2012) y Feijoo (1990) ahondaron en las condiciones de las trabajadoras, los paradigmas culturales y los deslizamientos entre diferentes actividades. Muy interesantes son las investigaciones sobre trabajadoras en distintos sectores: industria de la carne (Lobato, 1990), del dulce (Scheinkman, 2017), gráfica (Badoza, 1994), calzado (Kabat, 2007), vestido (Pascucci, 2007) y el trabajo rural (Roggio, 2001). Estos excelentes trabajos abordan, en mayor o menor medida, el problema de la desigualdad salarial, con los datos de que disponen. Sin embargo, queda un área de vacancia con respecto a series extensas de salarios.

\footnotetext{
${ }^{9}$ Algunos trabajos desarrollan la "hipótesis de concentración" de mujeres de ocupaciones determinadas; esto aumenta la oferta de mano de obra generando exceso de la misma y menores salarios (Bergmann, 1974). También en Macpherson y Hirsch (1995). Una crítica en Blau et al. (2002).

${ }^{10}$ Se debe mencionar que todo tipo de discriminación (o segregación) son elementos negativos, no sólo desde la perspectiva de género, sino en términos de eficiencia económica.
} 
De ese conjunto, nos interesa resaltar algunos, en particular los que ponen en cuestión el concepto mismo de "trabajo" al incluir no sólo las actividades remuneradas en el mercado, sino todas las actividades necesarias para llevar a cabo la reproducción de la vida. En consecuencia, como plantea Scheinkman (2019) se abre la posibilidad de repensar las pertenencias de clase a partir de la revitalización que proporcionan los actuales estudios de género. También permite pensar que la asociación entre el trabajo doméstico y las mujeres se debe a las relaciones sociales que buscan limitar la vida de las mujeres a su rol de madre (Nari y Lobato, 2004).

Particularmente sobre el trabajo doméstico, el trabajo de referencia es el de Cecilia Allemandi (2015), quien analiza en detalle el servicio doméstico en Buenos Aires entre fines del siglo XIX y principios del XX. Resulta interesante el funcionamiento de este sector, en particular las formas y costumbres de "contratación", así como la construcción de sentido al interior de los núcleos familiares donde desarrollaban sus tareas. El conjunto de tareas dejan ver una gran complejidad, claramente con predominio femenino (o "femenizado") ${ }^{11}$. Allemandi presenta una prospección de niveles salariales para las trabajadoras domésticas, para algunos años. Lo cual deja una vacancia para construir una serie de salarios extensa, que permita observar la evolución de las remuneraciones de las trabajadoras domésticas en el largo plazo.

\section{Metodología y fuentes}

Este trabajo adopta un enfoque cuantitativo; se busca analizar el nivel y la evolución de los salarios de las trabajadoras del servicio doméstico, en el contexto de la economía argentina, en la primera mitad del siglo XX. En este sentido, queda fuera de sus objetivos un análisis sobre las experiencias particulares y la forma en la que se desarrollaban estas tareas, que es analizado por Allemandi (2015).

Para la construcción de la serie de datos se tomaron en cuenta los trabajos de Allemandi (2015) y Newland y Cuesta (2017). Entre las diversas fuentes que analiza la primera se encuentra el periódico "La Prensa", a partir del cual formuló estimaciones sobre los salarios de las trabajadoras domésticas para algunos años. Por otro lado, Newland y Cuesta trabajan con el periódico “La Nación”, para el período 1936-1956. La serie de estos últimos se construyó con los pedidos realizados por los empleadores en los clasificados del diario para 3 días de Junio (en general, el 1, 15 y 30). Con esa masa de datos calculan la media simple anual. En este trabajo se utiliza el periódico "La Prensa", con la metodología de Newland y Cuesta. Se relevaron los datos de la sección "servicio doméstico pedido" (demanda) para los días 1, 15 y 30 de junio de cada año ${ }^{12}$.

\footnotetext{
${ }^{11}$ Por diferentes motivos, el trabajo de servicio doméstico estaba reservado, principalmente, para las mujeres, con la excepción de ciertas actividades asociadas a los varones, como la de "sereno" (quizá por la asociación del trabajo nocturno con el peligro o la ilegalidad, así como el paradigma de la domesticidad).

${ }^{12}$ En algunos años fue imposible recopilar la información de esas fechas, principalmente porque el soporte material de la fuente no estaba en condiciones. En esos casos se utilizó el día anterior o el siguiente, es decir, el 31 de mayo o el 2 de junio.
} 
Más allá de sus complicaciones, esta metodología resulta útil en un contexto en el cual no existen fuentes oficiales y los datos, en general, son sumamente escasos como consecuencia de las características particulares de este sector. Por ejemplo, por la fuerte informalidad que muchas veces deviene en relaciones que exceden el mero vínculo laboral.

No se utilizó la oferta de trabajo doméstico para estimar el salario de las trabajadoras (los anuncios de trabajo ofrecido) puesto que, dada la informalidad y la situación de precariedad de las empleadas domésticas, es difícil que lograran imponer sus aspiraciones salariales. En este sentido se considera mucho más pertinente tomar como referencia la demanda de trabajo, ya que potencialmente tiene más rigor dada su menor elasticidad con respecto al salario efectivo final.

Se calculó la media de la muestra obtenida y su respectivo desvío estándar. Con el fin de garantizar la representatividad, y teniendo en cuenta la presencia de ciertos datos anómalos, se decidió tomar para cada año un mínimo de 50 muestras, eliminado los extremos. De esta manera, se obtuvo una serie larga, continua y confiable de salarios de trabajadoras mayores de edad del servicio doméstico, de "mercado".

La serie de salarios nominales fue transformada en salarios reales, deflactando por el Índice de Precios al Consumidor (IPC), de Cuesta (2012a) y la Dirección Nacional de Estadísticas y Censos (1963).

La elección del periódico "La Prensa" se fundamenta en que se lo considera el periódico de época con mayor capacidad de brindar un proxy de un índice salarial doméstico lo suficientemente representativo. Con más puntualidad, al ahondar en el estudio de una relación de trabajo estrechamente relacionada a los sectores "acomodados", fue necesario encontrar una fuente que refleje el principal caudal de búsqueda sobre el sector mencionado. De esta manera, se consideró que el diario "La Prensa" era propio de un lector de clase alta, y/o en su defecto, de aquel que aspirara a formar parte de la élite económico-social.

En lo que respecta a la ubicación geográfica de las búsquedas, se observa que la mayor parte de los anuncios de los empleadores son de zonas aledañas a los barrios que se asociaban a los sectores urbanos de mayor capacidad económica. Se puede inducir que muchos de los departamentos/casas de esos empleadores se habían adaptado a lo largo de los años de distintas maneras, con el objetivo de incorporar la presencia de mucamas/

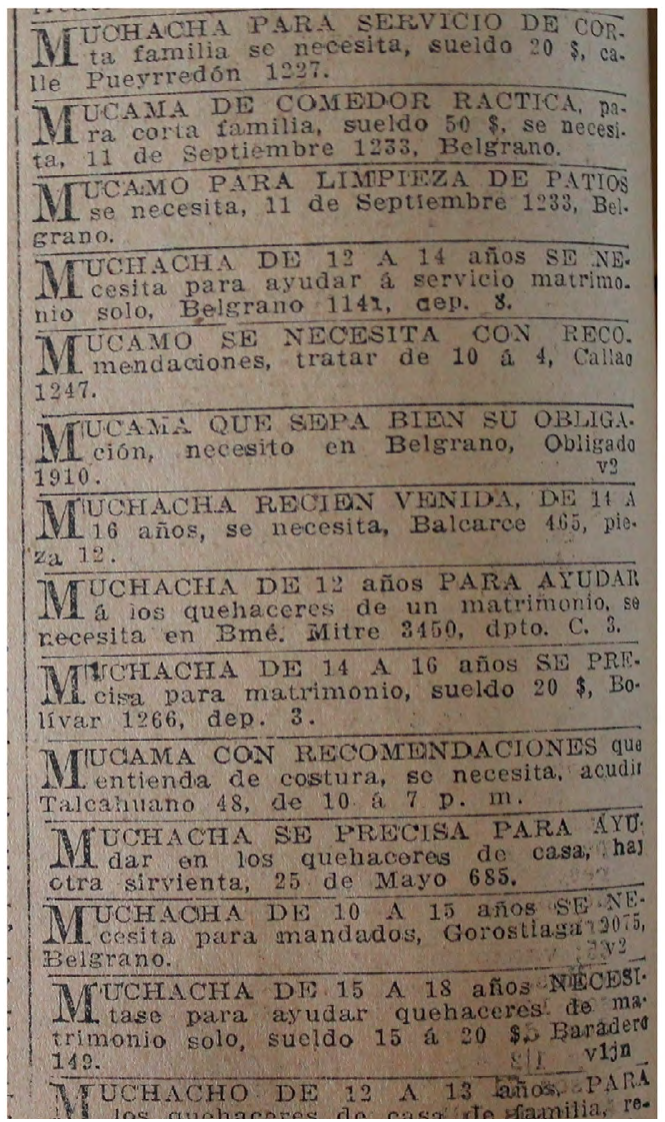

Fuente: Diario La prensa, 30 de junio de 1913. trabajadores", se pueden leer la demanda de trabajadoras domésticas. Es un ejemplo de la fuente utilizada para relevar los datos de este trabajo.
Imagen 1. 
sirvientas cama adentro (incorporación de entradas de servicio y pequeñas habitaciones ideadas para que la mujer se hospede como brazo familiar).

Se tomó como grupo de análisis a las empleadas "cama adentro", aquellas que residían en el domicilio de manera permanente. De todas maneras, se pudo observar que no todos los anuncios pertenecían a empleadas cama adentro; se pueden encontrar pedidos de trabajadoras que no dormían en el hogar del empleador, lo que no necesariamente implica una mayor o menor remuneración al trabajo. Es decir, no podemos encontrar una relación determinante entre empleadas cama adentro/cama afuera con respecto a mayor/menor salario. Posiblemente se pueda estar planteando un "trade-off" entre dos situaciones: la trabajadora "cama adentro" recibía un pago implícito, en forma de cama y comida, que el empleador podía usar como estrategia para justificar la reducción del salario, a manera de compensación. Así, terminaría por cumplir más horas, lo que resultaría en un mayor salario. En resumidas cuentas, se puede suponer que los resultados dependen de la relación laboral en cada caso, y que, dada la informalidad del sector, se muestren particularmente dispersos.

Otro aspecto a tener en cuenta es la diferenciación por "mucamas", "sirvientas" y "muchachas". La categorización de mucamas parece la más jerárquica de las tres en términos salariales. Por ejemplo, del análisis de los anuncios publicados en 1938 en "La Prensa", los días 1, 15 y 30 de junio, de un total de 113 búsquedas de trabajo a tiempo completo dentro de las tres categorías mencionadas, los 8 salarios más altos pertenecen a mucamas, mientras que a medida que disminuyen los salarios ofrecidos se empiezan a encontrar "sirvientas" y "muchachas" alternadamente. Esta diferencia podría explicarse teniendo en cuenta que el término "mucama" se asocia a trabajadoras experimentadas, que, en principio, percibirán un salario mayor. Por otro lado, los anuncios, no muestran diferencias entre las tareas y la remuneración de las "sirvientas" y las "muchachas". La distinción entre ambas posiblemente corresponda a imaginarios sociales antes que a diferencias laborales (Allemandi, 2015).

\section{Contexto económico y empleo}

Para dar un contexto y dimensionar adecuadamente las series, se presenta la evolución del PBI de Argentina, y del empleo y de la población de la ciudad de Buenos Aires.

La economía argentina en la primera mitad del siglo XX creció de manera sostenida y en gran magnitud (más de 5 veces). Obviamente, no estuvo exenta de algunos impactos, que son claros: la I Guerra Mundial y la crisis de 1930. Mucho menor fue el impacto de la II Guerra Mundial, y la crisis de 1950-52. De naturaleza local, esta hizo caer el producto, que se recuperó rápidamente al año siguiente (grafico 1).

Este intenso crecimiento de la actividad económica implicó un aumento de la producción; y, por ende, del empleo. En principio, se puede suponer que la demanda de trabajo se vio afectada.

Asimismo, la oferta y demanda de trabajo está en estrecha relación con la evolución de la población local. Se presenta a continuación la misma en el gráfico 2. 
Gráfico 1. PIB de Argentina (1900-1955). Base $100=1950$.

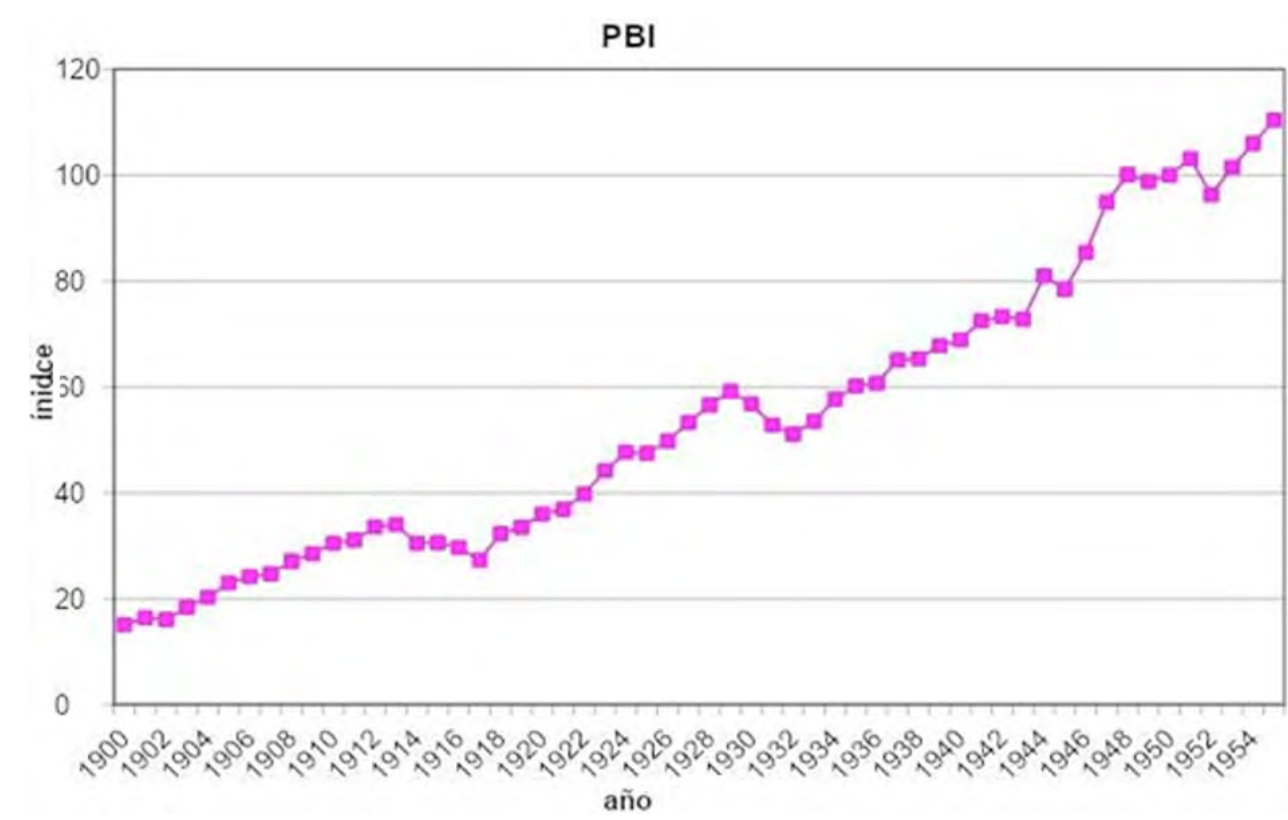

Fuente: CEPAL (1958).

Gráfico 2. Población de la ciudad de Buenos Aires (1904-1970) total y por sexo.

Población de la Ciudad de Buenos Aires (1904-1970)

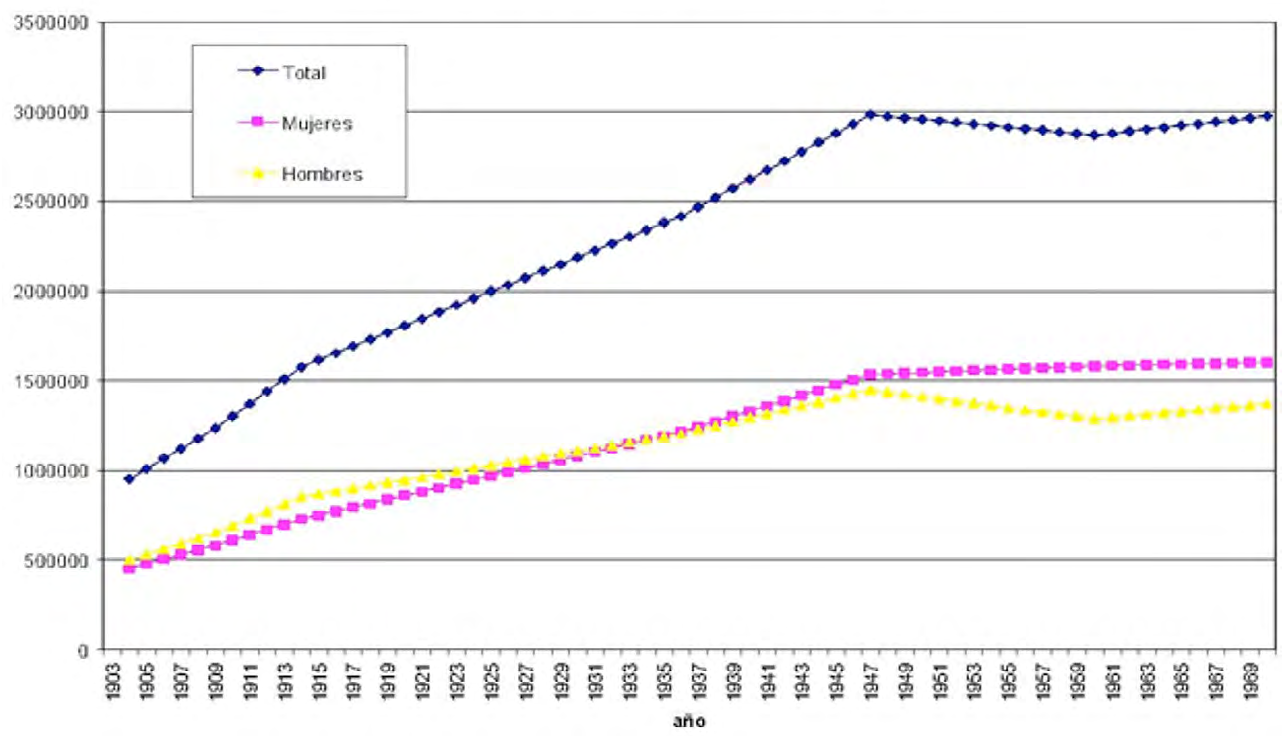

Fuente: Martínez (2019).

En conjunción con el crecimiento de la economía, aumentó la población de CABA. Se ve el impacto de la I Guerra Mundial y cómo desde entonces y hasta 1947 el crecimiento se suaviza; se estabiliza la población en menos de 3 millones de habitantes, en parte, como resultado de la expansión de la ocupación del espacio del conurbano de la ciudad, extramuros.

Resulta interesante observar la evolución de la población por sexo. La población masculina muestra un comportamiento muy similar a la población total, la tasa de masculinidad fue positiva hasta 1936. Esto es el resultado de la gran inmigración de las primeras 
tres décadas del siglo XX. Recién entre mediados de la década de 1920 y hasta mediados de la de 1940 cambia la tendencia; desde allí en adelante, la tasa de masculinidad se coloca en valores normales para una población estable, estabilizándose también la población total de la ciudad, en alrededor de tres millones de habitantes ${ }^{13}$.

La población femenina también creció, sin sufrir tanto el impacto de la I Guerra Mundial. Hacia 1930, cuando cambia la tasa de masculinidad, la población femenina supera a la masculina. También resulta interesante señalar que después de 1947, la población femenina se estabiliza, pero no cae (como le ocurre a la masculina) ${ }^{14}$.

Esto no está exento de impactos a nivel social y económico. Una información importante, que lamentablemente no está disponible para todo el período, es la ocupación y el desempleo. La cantidad de mujeres obreras en Buenos Aires se mantuvo estable durante muchos años, cayendo a mediados de la década de $1920^{15}$.

Tabla 1. Tasa de desempleo, empleo masculino, femenino y de menores en Buenos Aires en el sector industrial (1914-1927).

\begin{tabular}{|c|c|c|c|c|}
\hline \multirow{2}{*}{ Año } & \multicolumn{2}{|c|}{ Población ocupada en la industria } & \multirow{2}{*}{ \% de Desempleo } \\
\cline { 2 - 5 } & Hombres & Mujeres & Menores & 15,3 \\
\hline 1914 & 246636 & 72925 & 22324 & 18 \\
\hline 1915 & 242189 & 71685 & 24423 & 24,8 \\
\hline 1916 & 224289 & 66452 & 24058 & 30,1 \\
\hline 1917 & 209808 & 62300 & 22256 & 20,8 \\
\hline 1918 & 240170 & 71218 & 20832 & 17,5 \\
\hline 1919 & 252413 & 74786 & 25851 & 16,8 \\
\hline 1920 & 257690 & 76356 & 25568 & 18,2 \\
\hline 1921 & 258128 & 76486 & 25611 & 17,7 \\
\hline 1922 & 256046 & 75874 & 25406 & 15,1 \\
\hline 1923 & 274437 & 81145 & 27176 & 17,1 \\
\hline 1924 & 292821 & 86580 & 28996 & 16,5 \\
\hline 1925 & 290167 & 85600 & 32631 & 11,7 \\
\hline 1926 & 301823 & 91773 & 27625 & 9,2 \\
\hline 1927 & 350349 & 82917 & 28454 & 11,6 \\
\hline 1928 & 360859 & 85405 & 27885 & 15,6 \\
\hline 1929 & 353642 & 83696 & 27606 & \\
\hline 1930 & 350106 & 82859 & & \\
\hline
\end{tabular}

Fuente: elaboración propia a partir de Dirección General de Estadística Municipal (1925; 272) y Shipley (1977; 348).

A grandes rasgos, se denota el impacto del I Guerra Mundial. A nivel de género, se observa que en porcentajes las mujeres son el $21 \%$ de la población ocupada total entre 1914 y 1925. En 1926 alcanza el pico de 22\%, para luego caer al 18\% desde 1927 a 1930.

\footnotetext{
${ }^{13}$ Desde allí en adelante la población de la ciudad se estanca en esa cifra, dando lugar al crecimiento de los suburbios de la ciudad.

${ }^{14}$ ¿Es acaso una feminización de Ciudad de Buenos Aires?

${ }^{15}$ Lamentablemente no se dispone de datos de desempleo para los años anteriores. Y para los posteriores, no hay discriminación entre mano de obra masculina y femenina. Ver Cuesta y Cuk (2019).
} 
Esto está señalando evoluciones dispares de la ocupación de la mano de obra, que obviamente debió tener impacto en los salarios, y su capacidad de consumo. En una economía en crecimiento rápido y elevado, con una población urbana también en crecimiento, la demanda de servicios se incrementa.

Si se entiende que los salarios de las trabajadoras del servicio doméstico estaban en relación con el mercado (oferta y demanda), con lo cual el volumen era elástico a las fluctuaciones, vemos la evolución del PBI como proxy de la variación de capacidad adquisitiva de la demanda de trabajo (sectores medios y medios altos urbanos), así como de las variaciones en la cantidad de la oferta. Por otro lado, la evolución de la población puede ser un indicio de la evolución de la oferta de trabajo, en conjunción con los datos disponibles de empleo.

\section{Salario de las trabajadoras del servicio doméstico (1908-1956)}

En esta sección se presentan la evolución salario real de las trabajadoras del servicio doméstico a tiempo completo de empleadas mayores de edad, entre 1908 y 1956, en la ciudad de Buenos Aires (gráfico 3).

A grandes rasgos, en todo el período, el incremento en los salarios reales de este sector habría sido de un 20 \% (con máximo en 40\%). El mínimo habría estado en 1918, con una reducción de casi el $60 \%$ en términos reales.

Gráfico 3. Salario Real de las trabajadoras domésticas de Buenos Aires (1908-1956). Base 100=1908.

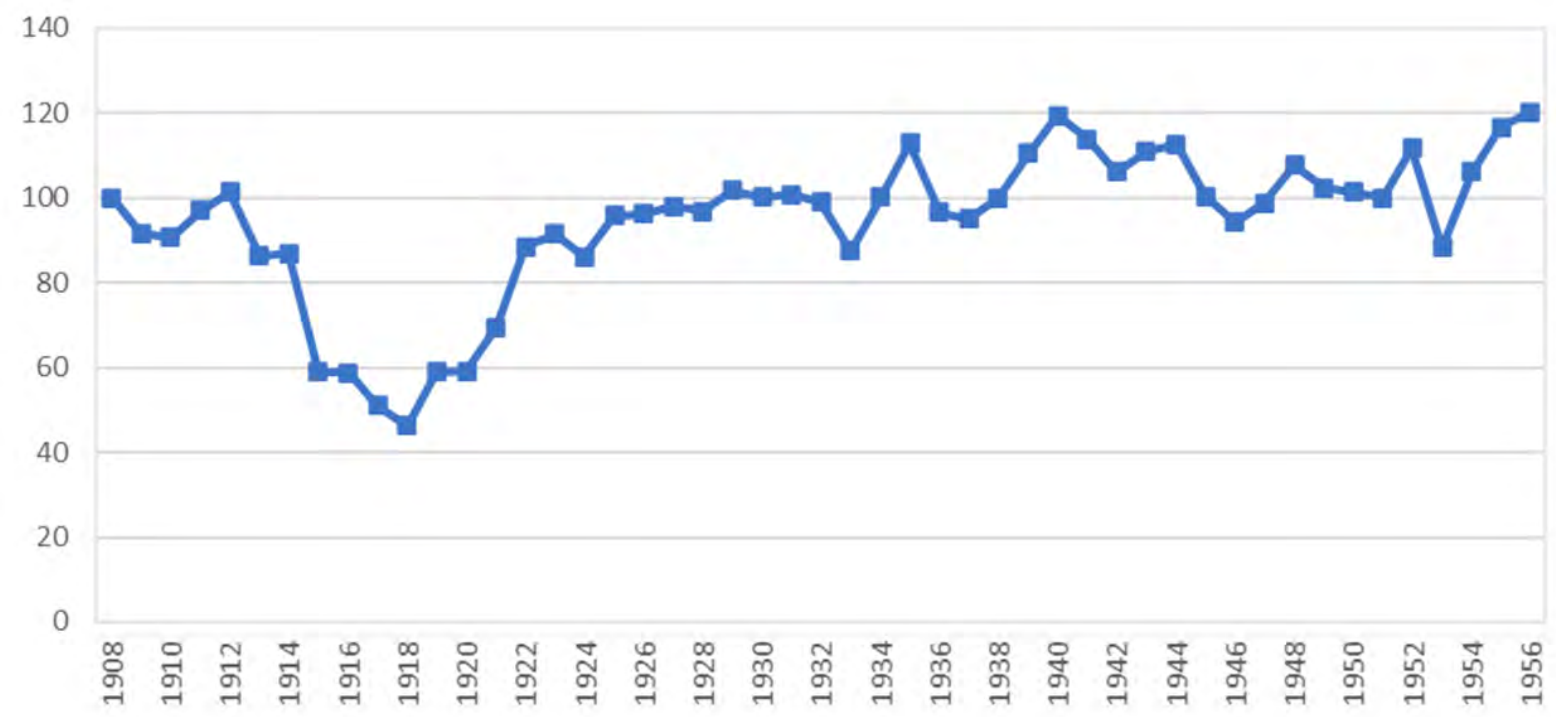

Fuente: Elaboración propia en base al periódico La Prensa y Newland y Cuesta (2017).

Se observa una fuerte caída entre 1912 y 1918, como resultado de la crisis de la I Guerra Mundial, que impactó en el PBI y en la ocupación general y femenina. Luego, se produjo una recuperación en el período 1918-1922 hasta alcanzar niveles levemente inferiores a los de 1912. Esto coincide con lo que se conoce de ocupación y desempleo, así 
como a la recuperación del PBI. A partir de 1922, la tendencia parece ser creciente, pero con un moderado estancamiento, fluctuando en aumentos (o descensos) anuales de no más del 10\%.

En la década de 1920 el salario real de las trabajadoras domésticas recuperó el poder adquisitivo de 1908, en un contexto de crecimiento del empleo y del PBI. De cualquier manera, se pueden caracterizar los años entre 1923 y 1946 como años de estabilidad del salario real en el sector doméstico.

Resulta interesante observar que, en la década de la crisis mundial de los años 30, el salario de las domésticas tiene una caída puntual en 1933, como el PBI argentino, pero que se recupera y supera los niveles de los años previos. Con la II Guerra Mundial, y con el estancamiento del PBI, crecen los salarios reales, llegando a un $20 \%$ más que el inicio de la serie. Este período coincide con el cambio en la población de la ciudad, que modifica la tendencia con respecto a la tasa de masculinidad.

Asimismo, si bien los salarios crecen durante el período, lo hacen en menor medida que el producto. De esta manera, se evidencia una caída del sector en la participación. Esto podría deberse al crecimiento de sectores con salarios e ingresos mayores.

Los años del peronismo (1946-55) impactaron en los salarios de las domésticas. Los salarios crecen en moderadamente en el período 1946-1952, caen considerablemente en el año 1953 y se recuperan entre 1954 y 1956. Desde una mirada impresionista, se observan indicios de correlación entre el PBI y el salario real de las trabajadoras domésticas, aunque es mucho más elástico el salario a la baja del PBI, que a la suba.

Esta relación puede deberse a que, junto al impacto que las recesiones tienen sobre los salarios, en el caso particular de las empleadas domésticas, se suma el crecimiento en la oferta de trabajo, al incorporarse mujeres al mercado laboral (principalmente de manera informal) frente a la caída de los ingresos del hogar.

Si se observa la evolución de la población femenina de la ciudad, en tanto proxy de la oferta de trabajo, parece no haber una correlación directa. Sin embargo, en conjunción con la evolución del empleo, se puede pensar que la caída en la demanda de mujeres en la industria puede tener relación directa con el aumento de la oferta de trabajadoras en el servicio doméstico; un efecto "desplazamiento". Más difícil es observar un sentido inverso, dado los paradigmas culturales de la época.

\section{Consideraciones finales}

En este trabajo se ha presentado y analizado la evolución de los salarios reales de las trabajadoras domésticas en la ciudad de Buenos Aires en la primera mitad del siglo XX. Esto cubre una vacancia en el tema, a partir de relevar nuevos datos y fuentes.

Entendiendo la evolución de las series en su contexto, pareciera haber una fuerte relación entre el comportamiento del salario de las trabajadoras del servicio doméstico y el PBI. Ascienden y descienden en los mismos momentos, pero pareciera ser una relación que se da con más fuerza en las recesiones que en las expansiones. Particularmente lla- 
mativa, es la caída que experimentan los salarios durante la Primera Guerra Mundial, muy superior a la que sufre producto.

Por otro lado, un factor que no pareciera ser muy relevante es el crecimiento de la población. Si bien es necesario un análisis más detallado, esto podría deberse a la confrontación entre dos efectos en direcciones opuestas: por un lado, el crecimiento poblacional, fundamentalmente de la población femenina, hace aumentar la mano de obra disponible, con lo cual se esperaría que los salarios tendieran a la baja. Por otro, la mayor cantidad de residencias y el proceso de ascenso social que experimentó parte de la sociedad hacen que las trabajadoras domésticas sean cada vez más requeridas. De esta manera, pareciera ser que estos efectos se compensan, sin afectar de forma considerable los salarios.

Otro elemento a considerar es hasta que punto la evolución de la actividad económica, y del empleo industrial de las mujeres, impacta por efecto desplazamiento en la oferta de trabajadoras domésticas. Esto, en parte, podría explicar la alta sensibilidad del salario frente a las crisis económicas.

Una explicación posible a la sensibilidad del salario frente a las crisis radica en la informalidad del sector, así como al "paradigma de la domesticidad", que posterga estas tareas, y, por consiguiente, la remuneración.

En este sentido, el ascenso de los salarios reales durante el período del peronismo (1946-55) es significativo. Si bien era un sector informal, se benefició directa y/o indirectamente de las políticas salariales peronistas. En el período 1946-1952 los salarios recuperan el poder adquisitivo perdido en los años previos. La caída del año 1953 evidencia el impacto de la crisis, pero para el período 1954-1956 vuelven a crecer. Sin lugar a dudas, este es un tema profundizar.

A partir del análisis de las fuentes, se observó una notable demanda de trabajadoras menores de edad, llegando incluso a buscar niñas de 11 años para realizar estos trabajos. Los salarios ofrecidos en estos anuncios eran notoriamente más bajos que los demás. Queda pendiente realizar un análisis centrándose en los salarios de las trabajadoras del servicio doméstico menores de edad.

No habría razones para suponer que el comportamiento de los salarios de estas actividades varíe sustancialmente respecto a la evolución de salarios en el conjunto del mercado laboral. En este sentido, la brecha salarial difícilmente sea una categoría central en el análisis de este mercado laboral puesto que en muy pocas actividades la presencia masculina emparejaba la femenina, como es el caso de los/as cocineros/as. Por el contrario, las desigualdades de género se evidencian al encargar estas tareas a las mujeres (iy a las niñas!), y en los bajos salarios de esta actividad en relación a otras de igual capacitación.

Sería interesante comparar los salarios de las trabajadoras domésticas con los salarios de trabajadoras de otras actividades. También establecer comparaciones con sectores masculinizados, como podrían ser los obreros de la construcción, con el objetivo de captar la diferencia en la remuneración como consecuencia de la discriminación de género ${ }^{16}$.

${ }^{16}$ Por ejemplo, Cuesta (2012b). 
Finalmente, otro camino a seguir es establecer comparaciones a escala regional, con los otros países de Latinoamérica, y luego a nivel global. De esta manera, se podría obtener una mirada de mayor amplitud que permita conocer los procesos de la región, así como las particularidades de cada país.

\section{Fuentes Primarias}

CEPAL. (1958). El desarrollo económico de la Argentina. Santiago de Chile: CEPAL.

Departamento Nacional del Trabajo. (1907-1920). Boletín del Departamento Nacional del Trabajo. Buenos Aires.

Departamento Nacional del Trabajo. (1918-1935). Crónica Mensual del Departamento Nacional del Trabajo. Buenos Aires.

Diario La Prensa. (1908-1957). Buenos Aires.

Dirección General de Estadística Municipal. (1925). Anuario Estadístico de la ciudad de Buenos Aires 1923. Buenos Aires: Briozzo Hermanos.

Dirección Nacional de Estadísticas y Censos. (1963). El Costo de nivel de vida en la Capital Federal. Buenos Aires.

MARTÍNEZ, RICARDO GABRIEL, RIAL, LUIS \& LEONE, JULIÁN (2019). Heterogeneidades sociales al interior de la Ciudad Autónoma de Buenos Aires. Madrid: Documento de trabajo. Instituto Universitario de Investigación en Estudios Latinoamericanos (IIELAT) Universidad de Alcalá.

MUZZILLI, CAROLINA (1913). El trabajo femenino. Boletín del Museo Social Argentino $n^{\circ}$ 15-16, pp. 60-82.

MUZZILLI, CAROLINA (1916). El trabajo femenino. Buenos Aires: Tall. Gráf. L.J.Rosso.

SHIPLEY, ROBERT EDWARD (1977). On the outside looking in: a social history or the porteño worker during the "golden age" of Argentine development, 1914-1930. Nueva Jersey: Phd Thesis, Rutgers University The State University of New Jersey (New Brunswick). Unión Patriótica Argentina. (1934). El trabajo femenino. Buenos Aires: Unión Patriótica Argentina.

\section{Bibliografía}

ALLEMANDI, CECILIA (2015). Sirvientes, criados y nodrizas. Una aproximación a las condiciones de vida y de trabajo en la Ciudad de Buenos Aires a partir del servicio doméstico (fines del siglo XIX- principios del XX). Buenos Aires: Tesis doctoral, Universidad de San Andrés.

BARRANCOS, DORA (2007). Mujeres en la sociedad argentina: una historia de cinco siglos. Buenos Aires: Sudamericana.

CAMOU, MARÍA MAGDALENA, MAUBRIGADES, SILVANA \& THORP, ROSEMARY (2016). Gender Inequalities and Development in Latin America During the Twentieth Century. Nueva York: Routledge. 
CUESTA, EDUARDO MARTÍN (2012a). Precios y salarios en Buenos Aires durante la gran expansión (1850-1914). Revista de Instituciones, Ideas y Mercados, No 56, Mayo, 2012, pp. 159-179.

CUESTA, EDUARDO MARTÍN (2012b). Precios, Salarios y diferencia de género en Argentina en la primera mitad del siglo XX. Cuadernos Koré, Universidad Carlos III de Madrid, número 7, otoño/invierno 2012, pp. 217-234.

CUESTA, EDUARDO MARTÍN \& CUK, MAURO NAHUEL (2019). Salarios en Argentina durante el siglo XX. Fuentes, estado del arte y propuestas de agenda. Ponencia presentada en las XVII Jornadas Interescuelas/Departamentos de Historia, organizadas por la Universidad Nacional de Catamarca, 2 al 5 de octubre, 2019.

D'ALESSANDRO, MERCEDES (2016). Economía feminista. Cómo construir una sociedad igualitaria (sin perder el glamour). Buenos Aires: Sudamericana.

ESPINO, ALMA (2011). Trabajo y género: un viejo tema, ¿nuevas miradas? Revista Nueva Sociedad, $N^{\circ} 232$, pp. 88-102.

ESQUIVEL, VALERIA (2007). Género y diferencias de salarios en Argentina. En MARTHA NOVICK, \& HÉCTOR PALOMINO (coords.), Estructura productiva y empleo (pp. 363392). Buenos Aires: Universidad Nacional de Quilmes.

FEIJO0, MARÍA DEL CARMEN (1982). La mujer en la historia argentina. Todo es Historia, $n^{\circ} 183$, pp. 8-16.

FEIJOO, MARÍA DEL CARMEN (1990). Las trabajadoras porteñas a comienzos del siglo. En DIEGO ARMUS (comp.), Mundo urbano y cultura popular. Estudios de Historia Social Argentina (pp. 281-311). Buenos Aires: Sudamericana.

FRANKEMA, EWOUT (2010). Reconstructing labor income shares in Argentina, Brazil and Mexico, 1870-2000. Revista de Historia Económica, no 28, pp. 343-374.

GOLDIN, CLAUDIA (1990). Understanding the Gender Gap: An Economic History of American Women. Nueva York: Oxford University Press.

GUY, DONNA (1981). Women, Peonage and Industrialization: Argentina 1810-1914. Latin American Research Review, Volumen XVI, $n^{\circ} 3$, pp. 64-89.

HAUSMANN, RICARDO; TYSON, LAURA \& ZAHIDI, SAADIA (2012). The Global Gender Gap Report 2012". Ginebra: Foro Económico Mundial.

İÑIGO Carrera, JUAN (2007). La formación económica de la sociedad argentina. Buenos Aires: Imago Mundi.

KABAT, MARINA (2007). Las mujeres en la industria argentina del calzado (1870-1940). En MARCELO LAGOS, MARÍA FLEITAS, \& MARÍA BOVI (comps.), A cien años del informe de Bialet Masse: el trabajo en la Argentina del siglo XX y albores del XXI (pp. 125-137). Jujuy: Universidad Nacional de Jujuy.

LOBATO, MIRTA (1990a). Mujeres en la fábrica. El caso de las obreras del frigorífico Armour, 1915-1969. Anuario del Instituto de Estudios Histórico Sociales, $n^{\circ}$ V, pp. 171-205.

LOBATO, MIRTA (1990b). Una visión del mundo del trabajo: el caso de los obreros de la industria frigorífica. Berisso, 1900-193. En DIEGO ARMUS (comp.), Mundo urbano y cultura popular. Estudios de Historia Social Argentina (pp. 313-317). Buenos Aires: Sudamericana. 
LOBATO, MIRTA (1993). Mujeres obreras, protesta y acción gremial en La Argentina: los casos de la industria frigorífica y textil en Berisso. En DORA BARRANCOS (comp.), Historia y Género (pp. 65-97). Buenos Aires: CEAL.

LOBATO, MIRTA (2000a). Entre la protección y la exclusión: discurso maternal y protección de la mujer obrera, argentina 1890-1934. En JUAN SURIANO (comp.), La cuestión social en Argentina, 1870-1943 (pp. 245-275). Buenos Aires: La colmena.

LOBATO, MIRTA (2000b). Lenguaje laboral y de género en el trabajo industrial. Primera mitad del siglo XX. En FERNANDA GIL LOZANO, VALERIA PITA, \& GABRIELA INI (coords.), Historia de las mujeres en la Argentina. Siglo XX (pp. 94-115). Buenos Aires: Taurus.

LOBATO, MIRTA (2001). La vida en las fábricas. Trabajo, protesta y política en una comunidad obrera, Berisso (1904-1970). Buenos Aires: Prometeo.

MARZONETTO, LUCÍA \& RODRÍGUEZ ENRÍQUEZ, CORINA (2015). El trabajo de cuidado remunerado. Estudio de las condiciones de empleo en la educación básica y en el trabajo en casas particulares. Buenos Aires: ELA-CIEPP-ADC. Serie de documentos de trabajo Políticas Públicas y Derecho al Cuidado Nro. 4.

MERCADO, MATILDE ALEJANDRA (1988). La primera ley de trabajo femenino. "La mujer obrera" (1890-1910). Buenos Aires: CEAL.

MOYANO LERENA, CARLOS (1957). El nivel de vida de los asalariados. Revista Panorama de la Economía Argentina, Buenos Aires, Estudio Llerena, agosto, pp. 90-100.

NAVARRO, MARISA \& WAINERMAN, CATALINA (1979). El trabajo de la mujer en la Argentina un análisis de las ideas dominantes en las primeras décadas del siglo XX. Buenos Aires: Cuaderno del CENEP.

NARI, MARCELA (1998). De la maldición al derecho. Notas sobre las mujeres en el mercado de trabajo. Buenos Aires, 1890-1940. En HILDA GARRIDO, \& MARÍA BRAVO (coords.), Temas de Mujeres. Perspectivas de Género. IV Jornadas de Historia de (pp. 139-155). Tucumán: Facultad de Filosofía y Letras. Universidad Nacional de Tucumán.

NEWLAND, CARLOS \& CUESTA, EDUARDO MARTIN (2017). "Peronismo y salarios reales. Otra mirada al período 1939-56". Investigaciones y Ensayos, Academia Nacional de la Historia, Buenos Aires, (enero-junio), no 64, pp. 75-98.

PASCUCCI, SILVINA (2007). Costureras, monjas y anarquistas; trabajo femenino, Iglesia y lucha de clases en la industria del vestido (Buenos Aires 1890-1940). Buenos Aires: Razón y revolución.

QUEIROLO, GRACIELA (2006). Mujeres que trabajan: una revisión historiográfica del trabajo femenino en la ciudad de Buenos Aires (1890-1940). Novo Topo. Revista de historia y pensamiento crítico $N^{\circ} 3$, pp. 29-48.

QUEIROLO, GRACIELA (2012). Mujeres en las oficinas. Las empleadas administrativas: entre la carrera matrimonial y la carrera laboral (Buenos Aires, 1920-1950). Revista do Departamento de História e do Programa de Pós-Graduação em História, vol. 16, núm. 2, mayo-agosto, 2012, pp. 417-444.

REYES CAMPO, NORA (2012). Women Wages and the Gender Gap during the Import Substitution Industrialization in Chile. Barcelona: Universidad de Barcelona. 
ROCCHI, FERNANDO (2000). Concentración de capital, concentración de mujeres. Industria y trabajo femenino en Buenos Aires, 1890-1930. En FERNANDA GIL LOZANO, VALERIA PITA, \& GABRIELA INI (coords.), Historia de las mujeres en la Argentina. Siglo XX (pp. 222-243). Buenos Aires: Taurus.

RODRÍGUEZ ENRÍQUEZ, CORINA (2017). Introducción a la Economía Feminista. Buenos Aires (Mimeo).

ROGGIO, PATRICIA (2001). Mujeres del campo: aproximación al estudio de la importancia del trabajo femenino en tareas rurales, Córdoba 1908-1947. Buenos Aires: Academia Nacional de la Historia. Obtenido de https://cehsegreti.org.ar/historia-social-2/ mesas\%20ponencias/MESA\%205/Ponencia\%20Patricia\%20ROGGIO.pdf

SCHEINKMAN, LUDMILA (2017). Trabajo femenino, masculino e infantil en la industria del dulce porteña en la primera mitad del siglo XX: experiencias laborales, protesta y vida cotidiana. Tesis de Doctorado, Facultad de Filosofía y Letras de la Universidad de Buenos Aires.

STOLKE, VERENA (2004). La mujer es puro cuento: la cultura del género. Revista Estudos Feministas, Florianópolis, 12 (2), pp. 77-105. 